\title{
Biological Mechanisms of Ageing
}

\author{
B. J. MERRY \\ Hull
}

\begin{abstract}
Summary
In a short review of the theories or proposed biological mechanisms of ageing it is necessary to be very selective in the ideas which are included or rejected. Although many theories of ageing are advanced in the literature a high proportion can be considered to be special cases of a previous hypothesis. No one individual theory satisfactorily explains the biological mechanism of ageing, indeed elements of many theories may be required to understand such a complex phenomena. Such a view has been formally expressed by Wright and Davison ${ }^{1}$ who consider that all theories represent too simplistic an approach to the understanding of ageing, a biochemical complexity which can only be resolved using system analysis techniques.
\end{abstract}

It has been a much repeated comment in the scientific literature that there are almost as many theories of ageing as there are investigators. Equally apparent is the lack of agreement that exists relating cause and effect in the assessment of biological mechanisms of ageing. Such a situation has arisen for two fundamental reasons. Firstly, and in spite of ageing being a property of most living organisms, this subject has attracted little scientific interest with the result that sound experimental data has been limited, resulting in an inevitable expansion in theoretical speculation. Secondly, ageing is multifaceted and hierarchical in its expression, affecting all components from the molecular level to the whole organism and is frequently confused with the symptoms of underlying pathology. Consequently many theories of ageing can be criticised for being merely an expression of some more fundamental change. ${ }^{2}$

It is possible to categorise the many disparate theories of ageing into genetic, nongenetic and physiological. The underlying assumption of genetic theories is that the life span of each species is determined by a developmental pro- gramme encoded in the DNA. Thus ageing may be envisaged to result from either the playing out of a pre-existing genetic programme with the possible activation of 'ageing genes' or it may result from damage to the process of information transfer from DNA to its metabolic expression in the cell. Nongenetic theories focus attention on the chemical or structural modification of cellular proteins with time after they have been synthesised, while physiological theories of ageing concentrate on the loss of communication between cells, tissues and organs placing particular emphasis on the regulatory role of the neuroendocrine system.

\section{Genome Based Theories}

\section{The Program Theory of Ageing}

Within a single species genetically determined differences in life span occur and in a study of inbred mouse strains Goodrick ${ }^{3}$ estimated that half the variance associated with longevity was due to genetic factors. Heterosis occurs when inbred strains are mated resulting in enhanced longevity but detailed genetic 
knowledge on the mechanisms of the inheritance of longevity is lacking. ${ }^{4}$

The central idea of the programmed theory is that ageing is the result of a sequence of events encoded in the genome just as the developmental sequence is controlled by gene expression. The finite ability of normal human and animal cells to replicate in vitro has provided the best evidence in support of this theory. ${ }^{2}$ Such an observation, however, may also be explained without invoking the concept of 'ageing genes'. While development from conception to adulthood is clearly programmed, ageing may merely be the momentum generated by the developmental programme which takes a species specific time to dissipate in a more or less random fashion.

Medawar ${ }^{5}$ has advanced the idea that ageing is an inevitable but mainly indirect consequence of natural selection. If it is not possible to eliminate a deleterious gene through natural selection, then it may be possible to postpone its time of expression. Consequently the effects of deleterious genes will, through the pressure of natural selection, be compressed towards the end of the life span. Williams ${ }^{6}$ has extended this argument to suggest that genes or gene clusters may have possible pleiotropic effects. If a gene had an unfavourable effect later in life which led to physiological deterioration and increased probability of death this gene would still be selected if the favourable effects early in life led to increased reproductive success.

It has been suggested ${ }^{7}$ that the idea of programmed ageing should be restricted to cases where there is a specific control of the onset of ageing by an identifiable metabolic process and a functional role for senescence can be demonstrated for that species, e.g. the rapid ageing and death of the Pacific salmon after spawning.

\section{The Somatic Mutation Theory}

The central concept is that if somatic cells accrue sufficient mutational damage in their DNA then physiological decrements characteristic of ageing will occur. Szilard ${ }^{8}$ suggested that ageing resulted from recessive mutations designated 'hits' occurring over the lifetime of the animal to inactivate a gene. The relatively low incidence of spontaneous mutations and the number of 'hits' required to achieve phenotypic expression argues against this idea. ${ }^{9}$ Moreover the predictions derived from this hypothesis have not been substantiated. It would follow that diploid organisms would live longer than haploid species but in the hymenopteran wasp Habrobracon, haploid and diploid males have similar life spans. Further arguments against the somatic mutation theory have been advanced by MaynardSmith ${ }^{10}$ and Alexander. ${ }^{11}$ The capacity for DNA repair in most cells would reduce the effect of such random mutations.

\section{The Error Theory}

In many respects this theory is a derivative of the somatic mutation theory since it is based on the idea that species differences to tolerate DNA damage may explain the variations in life span. The theory first proposed by Medvedev suggests that selective repetitions of certain genes (reiterative nucleotide sequences), would provide a protective mechanism against random damage in vital DNA sequences. The theory postulates that if a gene is repeated many times, damage to some copies of it will be less important than if the nucleotide sequence was unique. Therefore, the greater the proportion of the genome present in repeated sequence and the larger the number of repetitions for genes controlling general metabolism, the longer should be the life span. Medvedev ${ }^{12}$ has cited data for the number of repetitions of the genes for the major rRNA in bacteria, (5-10), Drosophila (100-130) and vertebrates (250-600) while Cutler ${ }^{13}$ although finding no correlation between reiterated DNA or ribosomal gene redundancy and longevity in mammals did observe that the average redundancy for genes transcribing mRNA in the brain of mice, cow and humans did correlate with longevity.

\section{Error Theory-Protein Synthesis}

Orgel proposed a model for biological ageing that was based on a decrease in the fidelity of protein synthesis. According to this theory ageing results from errors introduced in the process of information transfer from DNA through transcription and translation to the functional protein. Such errors are inevitable 
and most are likely to be unimportant but others may lead to further errors, e.g. misspecified RNA polymerases. Errors induced in the proteins controlling transcription and translation could amplify the original error to the point where the cell was no longer viable. Such a misspecified enzyme could produce a cascade effect called an 'error catastrophe'. This hypothesis has three major predictions. Firstly, degenerative changes observed with ageing should be primarily of cytoplasmic rather than nuclear origin. Secondly, abnormal proteins should be detectable in old cells and thirdly an experimentally induced increase in the error frequency of protein synthesis should accelerate the rate of ageing. The work of Wright and Hayflick ${ }^{14}$ utilising cell fusion techniques has questioned the first prediction. Hybrid fibroblasts with old and young cytoplasm and nuclei have suggested that cell senescence is not primarily due to cytoplasmic events. Despite the fact that altered proteins are now frequently demonstrated in old organisms this is not considered support for the Orgel hypothesis, the data strongly suggest that the changes observed in proteins are postsynthetic in nature. ${ }^{15}$

Direct measurements of fidelity of protein synthesis in aged animals are somewhat contradictory in their conclusions ${ }^{15}$ but attempts to induce accelerated ageing through an experimentally induced decrease in the fidelity of protein synthesis have so far failed. Induced errors in the fidelity of the protein synthesising systems of $E$. coli do not lead to an error catastrophe but to a stable level of errors which does not affect viability. ${ }^{15}$ Similarly when the synthesis of error containing proteins are induced in bacterial and animal cells by amino acid starvation the findings are contrary to the predictions of the error catastrophe theory (Fig. 1). ${ }^{16}$

The 'error catastrophe' theory of Orgel is just one of many mechanisms which relate cellular ageing with self-propagation of errors from DNA to protein synthesis (for review see ${ }^{17}$ ), while Rothstein and co-workers have formulated the hypothesis that altered enzymes in the cells of old animals can be explained by a slowing of protein turnover. ${ }^{15}$ It is suggested that an increase in the dwell time of cellular proteins would result in the subtle denaturation of some enzymes resulting in lowered specific activity.

\section{Nongenetic Theories}

\section{The Free Radical Theory}

Free radicals are highly reactive atoms or molecules in which an electron pair has been separated into two electrons that exhibit independence of motion,,$^{18}$ and are capable of initiating a chain reaction with stable molecules to generate more free radicals. The highly reactive nature of free radicals and their generation in all cells would lead to widespread damage particularly in the mitochondrial and microsomal membranes which contain oxidative enzymes such as monamine oxidase and microsome cytochrome oxidase. Further damage has been envisaged to occur by oxidative alterations in collagen, elastin, DNA, mucopolysaccharides, and lysosomes leading to the formation of ceroid and fluorescent age pigment. Free radical formation and oxidative activity have been proposed as the basis of certain pathological states such as fibrosis of arterioles and capillaries secondary to membrane damage. Implicit in the free radical concept is the accumulation of cellular damage and an inadequacy of cellular repair mechanisms to correct such damage. It is the stated belief of Harman, ${ }^{19}$ that 'the ageing process may be simply the sum of the deleterious free radical reactions going on continuously throughout the cells and tissues'. This biological mechanism has attracted much attention because of its universal application to all cells to explain random and diverse types of damage, and the proposal by Harman ${ }^{19}$ that free radicals are implicated in such pathologies as cancer, cardiovascular diseases, degenerative diseases of the central nervous system and the functional decline of the immune system.

Balin $^{20}$ has reviewed the data derived from experiments designed to test the free radical theory of ageing both in vivo and in vitro. Indirect evidence for the role of free radicals in ageing has been obtained from feeding antioxidants to laboratory animals. In a number of experiments with different strains of mice Harman $^{19}$ reported up to a 35 per cent increase in mean life expectancy but none of 


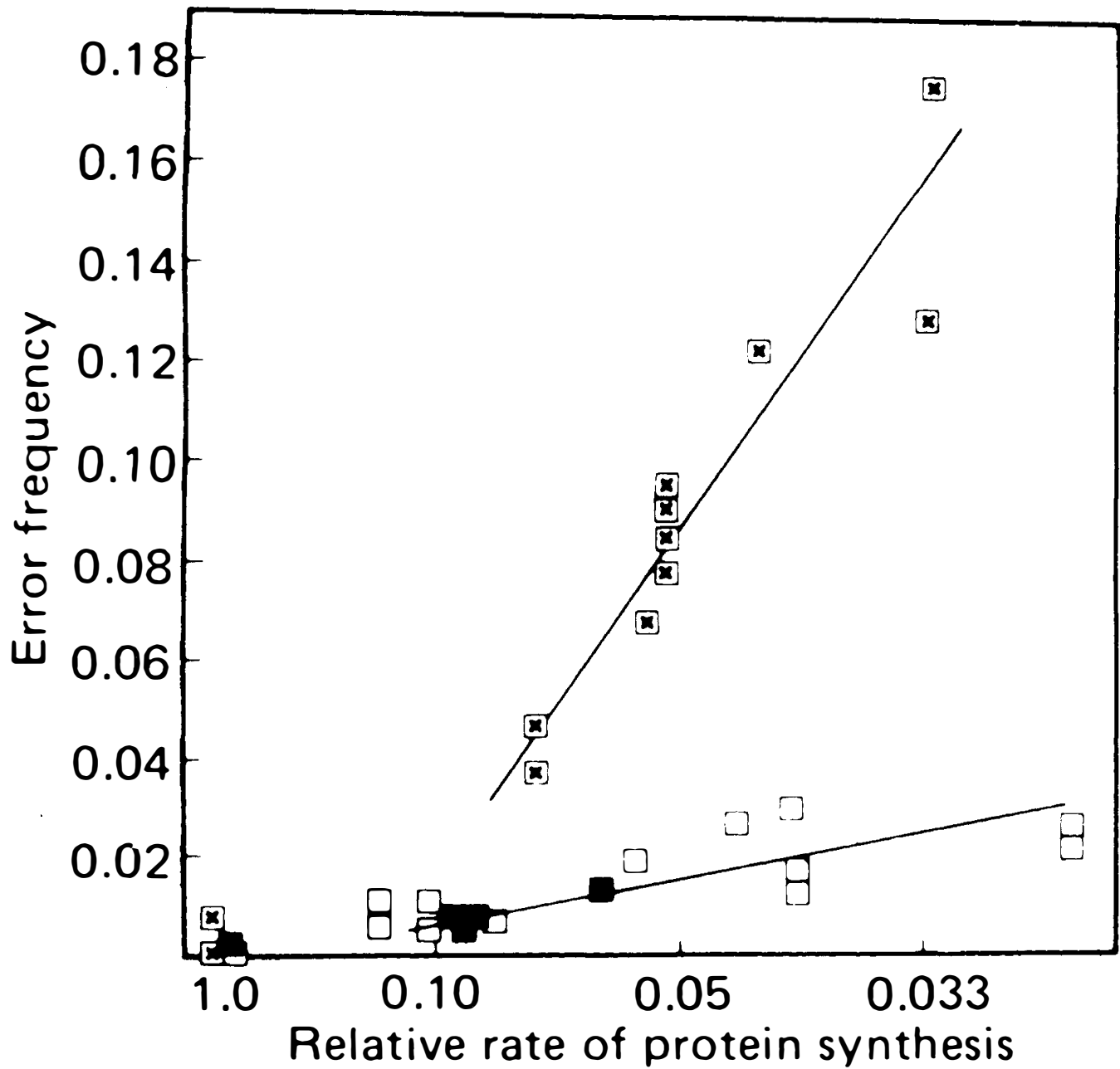

Fig. 1. Error frequencies are plotted against the reciprocal protein synthetic rate for early-passage WI38 ( $\square)$, latepassage WI38 (ם), and WI38-SV40 ( $\nabla)$ cells. Error frequencies represents the fraction of substituted histidine sites. Each point represents the error frequency determined by quantifying the scan of the autoradiogram from a single gel. (From Harley CB, et al. (1980). Reprinted with permission from the Proceedings of the National Academy of Sciences, USA, 77: p. 1889; Harley CB, Pollard JW, Chamberlain JW, Stanners CP, Goldstein S. Protein synthetic errors do not increase during aging of cultured human fibroblasts.)

the compounds tested extended the maximum life span. The antioxidants were most effective in increasing life expectancy of short lived strains of mice by delaying the development of specific pathologies, e.g. leukemia in AKR mice. Repeated experiments with the longer lived Swiss strain or the low tumour incidence strain LAF showed no effect of feeding antioxidants either on mean or maximum life span in either strain. Although Comfort ${ }^{4}$ demonstrated a significant increase in both mean and maximum life span of $\mathrm{C} 3 \mathrm{H}$ mice fed the anti- oxidant ethoxyquin he suggested alternative explanations for the effect were more plausible than that of molecular protection. Most importantly, in the majority of experiments where antioxidants are fed to rats and mice, a reduction in body weight is observed, a crucial observation since underfeeding is the classical way of increasing the maximum life span of laboratory species. ${ }^{21}$

While the role of free radicals as a fundamental cause of ageing is still uncertain, ${ }^{2}$ the demonstration by Cutler $^{22}$ that the ratio of 
repair or defence levels (superoxide dismutase) per specific metabolic rate is proportional to life span is noteworthy (Fig. 2).

\section{The Cross-Linkage Theory of Ageing}

Many common biological macromolecules will form covalent links between component parts of the same molecule or between molecules thereby modifying both structure and function. Molecular aggregation and immobilisation results with such complexes being resistant to catabolic processes. Central to the ideas of Bjorksten ${ }^{23}$ is that intracellular transport is impeded and essential information containing molecules (DNA), are damaged. Many compounds found in living tissue are known to be able to induce cross-linkages, of which free radicals are but one group, the free radical theory being a selected aspect of the cross-linkage theory. While cross-linkages in DNA and protein have been demonstrated over time, the majority of data in support of this theory derives from observations on the extracellular protein collagen. The loss of elasticity in many tissues but particularly the walls of blood vessels which results from such cross-linkage of collagen, will have detrimental effects on many organ systems and may be the first step in the ageing process. Criticisms of the theory are based on the lack of evidence that cross-linkage of intracellular proteins and DNA occurs in vivo at a level sufficient to account for the physiological decline characteristic of ageing. Hayflick ${ }^{2}$ has argued that the theory does not explain how the rate of molecular cross-linking is controlled to account for the wide range of life spans observed in animals.

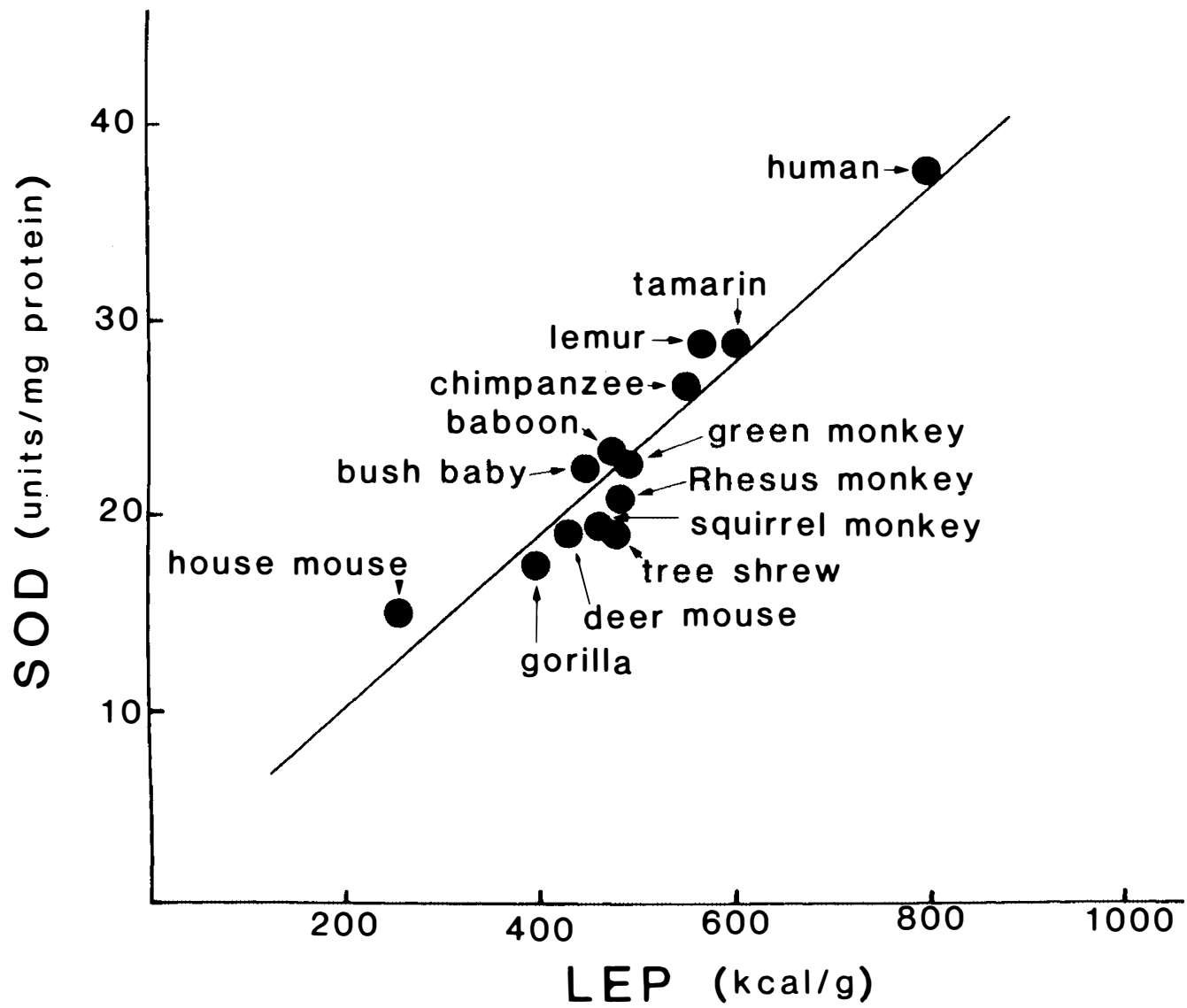

Fig. 2. Lifespan energy potential as function of superoxide dismutase (SOD) levels in liver. (From Cutler $R$ (1983). Reprinted with permission from Gerontology, 29: p. 119; Cutler R. Redundancy of information content in the genome of mammalian species as a protective mechanism determining aging rate. (C) 1983 Karger AG.) 
Table I. Tenth decile of survivorship in male mice congenic at the $H-2$ chromosomal region and on three different backgrounds

\begin{tabular}{|c|c|c|c|c|c|c|c|c|}
\hline \multirow{2}{*}{$\begin{array}{l}\text { Background } \\
\text { strain }\end{array}$} & \multirow{2}{*}{$\begin{array}{c}C 57 B L / 10 \\
H-2 \text { allele }\end{array}$} & \multicolumn{7}{|c|}{ 10th decile (weeks) } \\
\hline & & 1 & 2 & 3 & 4 & 5 & 6 & 7 \\
\hline $1 \mathrm{~B} 10 . \mathrm{AKM}$ & $m$ & $139 \pm 3.7$ & & & & & & \\
\hline $2 \mathrm{~B} 10 . \mathrm{Br} / \mathrm{Sg}$ & $k$ & $x$ & $149 \pm 1.1$ & & & & & \\
\hline 3 B10.PL & $u$ & $x$ & - & $153 \pm 1.7$ & & & & \\
\hline $4 \mathrm{~B} 10 . \mathrm{A} / \mathrm{Sg}$ & $a$ & $x$ & $x x$ & - & $154 \pm 0.4$ & & & \\
\hline 5 B10.D2/n & $d$ & $x$ & $x \times$ & - & - & $155 \pm 0.5$ & & \\
\hline $6 \mathrm{C} 57 \mathrm{BL} / 10$ & $b$ & $x$ & $x \times$ & - & $x$ & - & - & $155 \pm 0.4$ \\
\hline 7 B10.R111 & $r$ & $x \times$ & $x \times$ & $x \times$ & $x \times$ & $x \times$ & $x \times$ & $170 \pm 0.8$ \\
\hline A & & & & & & & & \\
\hline & & 8 & 9 & 10 & 11 & & & \\
\hline 8 A.BY & $b$ & $114 \pm 3.5$ & & & & & & \\
\hline 9 A.SW & $s$ & - & $123 \pm 6.6$ & & & & & \\
\hline 10 A.CA & $f$ & $x \times$ & - & $127 \pm 3.0$ & & & & \\
\hline $11 \mathrm{~A} / \mathrm{Wy}$ & $a$ & $x \times$ & $\times$ & $x \times$ & $134 \pm 1.7$ & & & \\
\hline $\mathrm{C} 3 \mathrm{H}$ & & & & & & & & \\
\hline $12 \mathrm{C} 3 \mathrm{H} / \mathrm{HeDi}$ & $k$ & $\begin{array}{c}12 \\
138+07\end{array}$ & 13 & 14 & & & & \\
\hline $13 \mathrm{C} 3 \mathrm{H} . \mathrm{JK}$ & $\kappa_{j}$ & $\begin{array}{c}138 \pm 0.7 \\
\times \times\end{array}$ & & & & & & \\
\hline 14 C3H.SW & $b$ & $\hat{x}$ & $\begin{array}{l}140 \pm 1.4 \\
\times x\end{array}$ & $150+12$ & & & & \\
\hline
\end{tabular}

$\times$ Indicates significant differences at the 0.05 and $\times \times$ at the 0.02 probability levels.

(Modified from Smith GS, and Walford RL (1977). Reprinted with permission from Nature 270: p. 728; Smith GS, and Walford RL, Influence of the main histocompatibility complex on ageing in mice. (C) 1977, Macmillan Press.)

\section{Accumulation Theories}

Impaired cell function with age has been ascribed to the accumulation of waste products within cells such as lipofuscin, a yellowgreen fluorescence product of lipid peroxidation involving the lysosomes. Such age pigment accumulates particularly in post-mitotic cells, such as neurones and cardiac muscle. The volume occupied by lipofuscin granules in human myocardium has been shown to increase linearly with age, with the large neurones of 100 year old individuals containing up to 75 per cent lipofuscin..$^{24}$ There is, however, no direct evidence that lipofuscin accumulation impedes cellular function while increased lipofuscin deposition in vitamin $\mathrm{E}$ deficient animals is not accompanied by accelerated ageing. Conversely vitamin E supplementation is not reported to prolong life span or decrease the accumulation of lipofuscin. ${ }^{24}$

\section{Physiological Theories}

The Immunological Theory of Ageing Walford has argued that much of observed ageing could represent increased autoim- munity and decreased heteroimmunity (for review see ${ }^{25}$ ). Maximum immune response occurs about the time of puberty in mammals followed by a subsequent decline to about 5-10 per cent of its maximum in old age. Concurrent with the decline in immune response, particularly in the $\mathrm{T}$ cell component is an increase in autoantibodies. Attention has been drawn to the association of the genetic control for the main histocompatibility complex and the genes regulating superoxide dismutase, mixed function oxidase and the level of cyclic nucleotides. It is proposed that the linkage of the genetic control for these factors on chromosome 6 in humans and 17 in mice is important in the control of ageing. In a study of 14 mouse strains genetically identical within a background strain except for the short chromosomal region carrying the $\mathrm{H}-2$ major histocompatibility complex Smith and Walford ${ }^{26}$ observed that within each genetic background significant differences were observed in survival to the tenth decile (Table I). It was concluded that a much greater uniformity of lifespan would have been expected 
within each congeneic set if the $\mathrm{H}-2$ allele did not have a significant effect on ageing.

The major criticism of the immunological theory of ageing is the lack of applicability to organisms that do not have an immune system similar to that observed in the vertebrates. Secondly, the immune system is regulated by hormonal control and therefore gene expression and immunological dysfunction may be initiated by the neuroendocrine system.

\section{The Neuroendocrine Theory}

It has been proposed that the process of ageing and senescence may be under neural and endocrine control in a manner similar to development, growth and maturation. ${ }^{27}$ Consequently this theory may be viewed as an organ based extension of the programmed theory of ageing for underpinning neuroendocrine regulation of ageing is the concept of an 'ageing clock', usually located in the hypothalamus. The neuroendocrine theory is a generic term used to describe a number of hypotheses which invoke such ideas as the disregulation of neuroendocrine control through neuron loss, neurotransmitter deficiencies, stress, hypopituitarism, hypothyroidism, or disruption of feedback sensitivity. A breakdown in the neuroendocrine central regulatory control would, it is argued, have widespead peripheral consequences leading eventually to overt pathology. ${ }^{27}$

The neuroendocrine theories of ageing can be criticised for not having universal applicability, in a manner not dissimilar to arguments used against the immune theory of ageing. Neither does this type of theory explain the initiating event at the molecular level which so disrupts neuroendocrine coordination with time. No data are available which clearly demonstrate that changes in the neuroendocrine system are the cause rather than the effect of ageing.

\footnotetext{
References

${ }^{1}$ Wright BE, Davison PF: Mechanisms of development and aging. Mech. Aging Dev. 1980; 12: 21319.

${ }^{2}$ Hayflick L: Theories of biological aging. Exp. Geront. 1985; 20: 145-59.

${ }^{3}$ Goodrick CL: Life-span and the inheritance of lon-
}

gevity of inbred mice. J. Gerontol. $1975 ; 30: 257-$ 63.

${ }^{4}$ Comfort A: The biology of senescence. 3rd Ed. London: Churchill Livingstone 1979.

${ }^{5}$ Medawar PB: The definition and measurement of senescence. In: Wolstenholme GEW, Cameron MP, eds. Ciba Foundation Colloquia on Ageing: I. General Aspects. London: Churchill 1955; 4-15.

${ }^{6}$ Williams GC: Pleiotropy, natural selection and the evolution of senescence. Evolution 1957; 11: 398-411.

${ }^{7}$ Sacher GA: Evolutionary theory in gerontology. Perspect. Biol. Med. 1982; 25: 339-53.

${ }^{8}$ Szilard L: On the nature of the ageing process. Proc. Natn. Acad. Sci. USA 1959; 45: 30-45.

${ }^{9}$ Shock NW: Biological theories of ageing. In: Florini JR, ed. CRC Handbook of Biochemistry in Aging. Florida: CRC Press Inc. 1981; 271-82.

${ }^{10}$ Maynard-Smith J: The causes of ageing. Proc. $R$. Soc. Lond. [Biol.] 1962; 157: 115-27.

${ }^{11}$ Alexander P: Is there a relationship between aging, the shortening of life-span by radiation and the induction of somatic mutation? In: Shock NW, ed. Perspectives in Experimental Gerontology. Illinois: Ch. C. Thomas 1966; 266-79.

${ }^{12}$ Medvedev ZhA: Repetition of molecular-genetic information as a possible factor in evolutionary changes in life span. Exp. Geront. 1972; 7: 22738.

${ }^{13}$ Cutler R: Redundancy of information content in the genome of mammalian species as a protective mechanism determining aging rate. Mech. Ageing Dev. 1974; 2: 381-408.

${ }^{14}$ Wright WE, Hayflick L: Contributions of cytoplasmic factors to in vitro cellular senescence. Fedn. Proc. Fedn. Am. Socs. exp. Biol. 1975; 34: 76-9.

${ }^{15}$ Rothstein M: Posttranslational alteration of proteins. In: Florini JE, ed. CRC Handbook of Biochemistry in Aging. Florida: CRC Press Inc. 1981; 103-11.

${ }^{16}$ Harley CB, Pollard JW, Chamberlain JW, Stanners $\mathrm{CP}$, Goldstein S: Protein synthetic errors do not increase during aging of cultured human fibroblasts. Proc. Natl. Acad. Sci. USA 1980; 77: 1885-9.

${ }^{17}$ Medvedev ZhA: The role of infidelity of transfer of information for the accumulation of age changes in differentiated cells. Mech. Ageing Dev. 1980; 14: $1-14$.

18 Pryor WA: Free radical reactions and their importance in biochemical systems. Fedn. Proc. Fedn. Am. Socs. exp. Biol. 1973; 32: 1862-9.

${ }^{19}$ Harman D: The aging process. Proc. Natl. Acad. Sci. USA 1981; 78: 7124-8.

${ }^{20}$ Balin AK: Testing the free radical theory of ageing. In: Adelman RC, Roth GS, eds. Testing the Theories of Aging. Florida: CRC Press Inc. 1982; 137-82.

${ }^{21}$ Holehan AM, Merry BJ: The experimental manipulation of ageing by diet. Biol. Rev. 1986; 61: 329-68. 
${ }^{22}$ Cutler RG: Superoxide dismutase, longevity and specific metabolic rate. Gerontology 1983; 29: $113-20$.

${ }^{23}$ Bjorksten J: Crosslinkage and the aging process. In: Rockstein M, ed. Theoretical Aspects of Aging. London: Academic Press Inc. 1974; 43-59.

${ }^{24}$ Strehler BL: Time, Cells and Aging. 2nd Ed. New York: Academic Press Inc. 1977.

${ }^{25}$ Walford RL, Weindruch RH, Gottesman SRS, Tam
CF: The immunopathology of aging. In: Eisdorfer C, ed. Annual review of Gerontology and Geriatrics. Vol. 2. New York: Springer 1981; 3-48.

${ }^{26}$ Smith GS, Walford RL: Influence of the main histocompatibility complex on ageing in mice. Nature 1977; 270: 727-29.

${ }^{27}$ Everitt AV: The neuroendocrine system and aging. Gerontology 1980; 26: 108-19. 